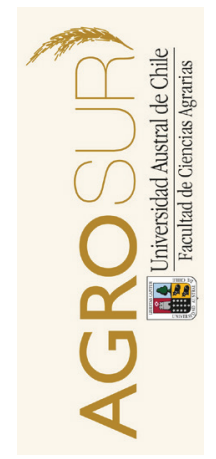

A R T I C L E IN F O

\begin{tabular}{l} 
A R T I C L E I N F O \\
\hline Article history: \\
Received 09.08.14 \\
Accepted 20.02.15 \\
\hline Keywords: \\
Finance \\
Food production \\
Fishing \\
Export \\
Aquaculture \\
\hline Case Study, \\
Agrarian Economy \\
\hline *Corresponding author: \\
Sandra Alfaro \\
E-mail address: \\
p.alfarosandra@hotmail.com
\end{tabular}

\section{Sector pesquero-acuícola en México y Chile: estudio de caso comparativo para reflexionar respecto de su internalización}

\author{
Fisheries-aquaculture sector in Mexico and Chile: \\ comparative case study to reflect on their internalization
}

\author{
Alfaro, S.B. ${ }^{a *}$, Quintero, M. L ${ }^{b}$. \\ ${ }^{a}$ Lic. Comercio Internacional, Universidad Autónoma del Estado de México (UAEM) \\ ${ }^{b}$ Unidad Académica Profesional Nezahualcóyotl, UAEM, México.
}

\begin{abstract}
A B S T R A C T
The aim of this paper is to compare the international economic importance of the fisheriesaquaculture sector in Mexico and Chile; highlighting the contributions in food production, foreign exchange earnings from exports and social role of the jobs created. This is because both Mexico and Chile have an adequate geographic location, sufficient water resources, quality workforce and experience earned by entrepreneurs, technicians and academics for decades, as well as accumulated fixed capital in fleet, ports, industries and marketing activities; which along with a neoliberal economic policy allow to have an exploitable and profitable aquaculture fisheries sector, with ample opportunities for economic growth. To achieve the stated objective the comparative method was applied, by explaining what parts make up the fishing-aquaculture performance of each sector in these countries, which elements influenced their behavior and problems; to finally join the heterogeneous elements of this phenomenon by the synthetic method in order to analyze the behavior of the observed object, and extract the most important elements to generate solid reflection on the subject.
\end{abstract}

\title{
RESUMEN
}

El objetivo de este artículo es comparar la importancia económica internacional del sector pesquero-acuícola en México y en Chile; resaltando las aportaciones en la producción de alimentos, la obtención de divisas por concepto de exportaciones y la función social en los empleos generados. Lo anterior se debe a que tanto México como Chile tienen una adecuada ubicación geográfica, suficientes recursos acuáticos, calidad en la fuerza de trabajo y experiencia ganada por empresarios, técnicos y académicos durante décadas, así como capital fijo acumulado en flota, puertos, industrias y actividades de mercadeo; lo que permite junto con una política económica de tipo neoliberal contar con un sector pesquero acuícola explotable y redituable, con amplias oportunidades de crecimiento económico. Para alcanzar el objetivo señalado, se aplicó el método comparativo, ya que primeramente se explicó que partes componen el funcionamiento del sector pesquero-acuícola de cada uno de estos países, que elementos influían en su comportamiento y problemática; para posteriormente por el método sintético unir los elementos heterogéneos de dicho fenómeno con el fin de analizar el comportamiento del objeto observado, y extraer los elementos más importantes para generar reflexión sólida sobre el tema.

Palabras clave: Financiamiento, producción de alimentos, pesca, exportación, acuacultura.

\section{INTRODUCCIÓN}

El objetivo de este artículo es comparar la importancia económica internacional del sector pesqueroacuícola en México y en Chile; resaltando las aportaciones de este sector en la producción de alimentos, la obtención de divisas por concepto de exportaciones; pero también cubriendo socialmente, la generación de empleos. Lo que posiciona a esta actividad en un papel estratégico internacional para el desarrollo económico-social de México y Chile; por lo que es necesario reflexionar algunas propuestas de recomendación para estar en mejores condiciones y permanencia en el mercado. Los procesos a desarrollar en dicho artículo tendrán un enfoque cuantitativo y de carácter interpretativo, con el fin de generar posibles alternativas para potencializar el crecimiento de la actividad pesquera. 
México tiene una gran riqueza natural en sus regiones oceánica y costera, lo que se debe a su singular posición geográfica intertropical. El dinamismo de la zona costera es producto de la interfase del continente, el océano y la atmósfera, donde tienen lugar diversas actividades económicas de importancia nacional e incluso mundial, como son la pesca y la acuicultura, la extracción de hidrocarburos y minerales y la transportación marítima y el turismo, por citar algunas (Lara-Lara et al., 2008).

Por su parte, Chile ha sido y es un actor fundamental en la creación y desarrollo del derecho internacional del mar; históricamente los pescadores artesanales en su mayoría no han sido contemplados en las propuestas de políticas legislativas-económicas, ni por las políticas públicas. En la década de los 90 se realizaron diversos cambios en materia de política económica, que modificaron las condiciones socio-culturales de los pescadores artesanales, ya que se fomentó la privatización de los recursos pesqueros. Lo que ha generado entre otras cosas la continua pérdida de fuentes de trabajo de los pescadores artesanales pasando a manos de grandes inversionistas (Gacitúa y Pizarro, 2007).

Lo expuesto nos lleva a cuestionar la siguiente problemática: ¿Qué acciones se deben llevar a cabo para aprovechar e impulsar el sector pesquero-acuícola en estos dos países, para tener una mayor presencia en el mercado internacional?; para contestar a esta pregunta consideramos que se tiene que realizar un diagnóstico de cómo se encuentra esta actividad para analizar y reflexionar sobre el papel que tiene el financiamiento en la actividad pesquera, con el objetivo de saber en qué rubros reinvertir en: infraestructura, capacitación, tecnología y crecer con rentabilidad y sustentabilidad; lo que incrementaría la propensión a exportar que la de importar, para alcanzar mayor crecimiento económico del país, generar recursos y empleos para ambos países. Lo anterior sustentado en la elaboración e implementación de políticas públicas que apoyen y desarrollen este sector, dando las facilidades de asesoramiento y capacitación de exportación, mercado y comercialización de los productos pesqueros de acuerdo a las necesidades y características específicas de los grupos productores en cada uno de estos países.

\section{MATERIALES Y MÉTODOS}

Esta investigación se enriquece con el análisis de datos y estadísticas de la Secretaría de Agricultura, Ganadería, Desarrollo Rural, Pesca y Alimentación (SAGARPA), Secretaría de Economía (SE), Organización de las Naciones Unidas para la Alimentación y la Agricultura (FAO), Comisión Nacional de Pesca y Acuacultura (CONAPESCA), Organización para la Cooperación y Desarrollo Económicos (OCDE), Instituto Nacional de Estadística y Geografía (INEGI), así como información de dependencias chilenas como la Subsecretaría de Pesca y Acuacultura, el Instituto de Fomento Pesquero y el Servicio Nacional de Pesca, entre otros.

El desarrollo del presente artículo se divide en cuatro partes. La primera hace un análisis de las teorías de financiamiento y desarrollo tecnológico, se reflexiona sobre el enfoque instrumental, el cognitivo y el sistémico, los cuales nos aportan un sustento teórico importante para los fines de la investigación. En la segunda parte se ofrece un panorama de los antecedentes y situación actual de la actividad pesquera-acuícola en México resaltando el volumen y estructura de la producción utilizando tablas comparativas de los Litorales del Pacífico, del Golfo y Caribe y, de las Aguas continentales de los últimos años, donde se considera el consumo humano directo e indirecto, y uso industrial, así como la balanza comercial y la importancia socio cultural y alimentaria de este sector; también se analiza el medio ambiente, infraestructura, tecnología y financiamiento. La tercera parte presenta información específica para el caso de Chile, donde se identifica entre otras cosas, la estructura de su producción, balanza comercial, empleo, tecnología. Finalmente, la cuarta parte, termina con la discusión y alternativas de reflexión sobre las tareas pendientes que tendrá que afrontar la actividad pesquera-acuícola en México y en Chile para el crecimiento competitivo internacional.

\section{DESARROLLO}

\section{Enfoque teórico: el financiamiento y el desarrollo tecnológico}

Para entender el actual funcionamiento del sector pesquero acuícola de México y Chile, la teoría del financiamiento y la teoría del desarrollo tecnológico dan aportaciones para explicar del proceso de evolución, innovación y transformación tecnológica que ha tenido este sector, a partir fundamentalmente del financiamiento y la inversión.

En este sentido, Modigliani y Miller (1958), con su Teoría de Financiamiento, interpretaron los equilibrios entre los costos y las ventajas del endeudamiento que una empresa o un sector económico pueden poseer; lo que permite fundamentar el valor del apalancamiento, la amortización de pasivos a plazos, que se utilizarán para acrecentar los niveles de capital en la empresa, y que serán aplicados en diferentes operaciones económicas, de índole operativa, financiera u otra, con el fin de elevar dicho nivel al máximo posible.

Esto da como consecuencia que una empresa podrá hacer frente a sus planes de inversión mediante el empuje inicial provisto por la inyección de recursos, suministrado por fuentes de dinero internas o externas. Las fuentes de financiamiento de las empresas son herramientas claves para proporcionar la fortaleza económica de las mismas y su competitividad en el tiempo. 
En relación a la teoría de Modigliani y Miller (1958), Singh y Hamid (1992), afirman que las grandes empresas de los países desarrollados se financian con una elevada proporción de fondos propios, en contraste con las de América Latina, que se basan más en emisiones de deudas y acciones. Debido a esto podemos interpretar que la aplicación del financiamiento se equilibra en relación a las necesidades presentadas por dichas empresas. Para Gitman (2003) el financiamiento a corto plazo utiliza ciertos activos como garantía, no así el financiamiento a corto plazo sin garantía. Pueden ser internas y externas a la empresa, y vienen definidas por las instituciones nacionales (públicas y privadas) e internacionales que otorgan recursos económicos.

Según Montoya (2004), Schumpeter compartía la opinión de Marx de que los procesos económicos son orgánicos y que el cambio surge desde dentro del sistema (proceso endógeno) y no desde fuera. De Walras tomó la noción de empresario, pero en lugar de su figura pasiva del sistema de equilibrio general, Schumpeter la sustituyó por un agente activo del progreso económico. Para Schumpeter, la fuerza fundamental, que mueve la producción capitalista, y al sistema como un todo, y que es la causante de la dinámica de transformación constante, es el proceso de innovación tecnológica. En ese contexto, el mismo autor indicaba que lo importante son las innovaciones radicales, aquellas capaces de provocar cambios "revolucionarios", transformaciones decisivas en la sociedad y en la economía (Montoya, 2004).

Shumpeter (1978) hace referencia al desarrollo económico ya que parte de dos conceptos fundamentales: la innovación como causa del desarrollo y el empresario innovador como propiciador de los procesos de innovación. El análisis de Shumpeter (1978) considera el proceso de producción como una combinación de fuerzas productivas, que están compuestas por fuerzas materiales y fuerzas inmateriales. Las fuerzas materiales son (trabajo, tierra y capital -"medios de producción producidos"). Las fuerzas inmateriales las componen los "hechos técnicos" y los "hechos de organización social", que, al igual que los factores materiales, también condicionan la naturaleza y el nivel del desarrollo económico. Los efectos de los cambios tecnológicos y sociales (tecnología, innovación y ambiente socio-cultural), ejercen un impacto más decisivo y más dinámico; por esta razón, estos factores inmateriales fueron denominados "fuerzas o factores del desenvolvimiento económico o evolución económica" (Shumpeter, 1978).

Por otra parte, continuando con el enfoque tecnológico, según Osorio (2002) autores más recientes como Quintanilla (1988) mencionan que, las grandes orientaciones o enfoques en las teorías sobre la técnica y la tecnología, pueden ser agrupadas en tres apartados: la orientación instrumental, la cognitiva y la sistemática. Coincide con Mitcham (1994) sobre las diferentes formas de manifestación de la tecnología: como cono- cimiento, como actividad (producción, uso), y como objetos (artefactos).

El enfoque instrumental o artefactual, se refiere la concepción artefactual o instrumentista de la tecnología; se considera que las tecnologías son simples herramientas o artefactos construidos para una diversidad de tareas. Este enfoque explica que se generan cambios en los individuos en su vida profesional, en su vida privada, y en su psiquismo (Osorio, 2002).

El enfoque cognitivo, plantea una distinción radical entre técnica y tecnología: al ser la tecnología producto de la aplicación de la ciencia, la técnica solo comprendería experticias que se logran por la actividad empírica, sin ayuda del conocimiento científico. El factor fundamental del desarrollo tecnológico sería la invención, la investigación y desarrollo (Osorio, 2002).

Finalmente el enfoque sistémico, retoma la noción de sistema para definir a la tecnología, como sistemas de acciones intencionalmente orientados a la transformación de objetos concretos, para conseguir de forma eficiente un resultado valioso. Desde esta perspectiva, el factor fundamental del desarrollo tecnológico sería la innovación social y cultural, la cual involucra no solamente a las tradicionales referencias del mercado, sino también a los aspectos organizativos, y el ámbito de los valores y de la cultura de los sistemas socio-tecnológico.

Como vemos la teoría del desarrollo económico fundamentada en los procesos de innovación y crecimiento tecnológico y en el cambio socio-cultural es una herramienta fundamental para entender como las empresas dedicadas a la actividad pesquera acuícola deben de introducir y perfeccionar el uso de tecnología para permanecer en el mercado. Por lo tanto, a partir de estos enfoques teóricos podemos retomar por un lado, la importancia que tiene contar con suficiente financiamiento para impulsar una actividad productiva, y que este financiamiento tiene que ser minuciosamente canalizado a las diferentes fases del proceso de producción, considerando la planeación de la inversión tanto en el corto, mediano y largo plazo; dando prioridad a las esferas que requieren de mayor inversión; y por el otro, resaltar el impulso al crecimiento tecnológico dentro de la actividad pesquera acuícola como elemento a considerar para detonar un mayor crecimiento y competitividad a nivel internacional. De ahí la relevancia de retomar las aportaciones planteadas por Schumpeter sobre el papel estratégico que tiene el empresario como generador de ideas e innovaciones que permitan la creación de tecnología. Asimismo, las aportaciones teóricas del enfoque instrumental, el cognitivo y el sistémico también arrojan categorías importantes para explicar cómo la evolución y desarrollo actual del sector pesquero acuícola en los países de estudio son resultado del conocimiento técnico, sustentado en una práctica social que toma en cuenta aspectos físicos, culturales y organizacionales. 


\section{Antecedentes y situación actual de la actividad pesquera-acuícola}

La pesca, es una actividad humana muy antigua, puede ser pesca tradicional o también llamada pesca industrial o comercial; para lo cual se aplican técnicas con el propósito de capturar por medio de embarcaciones animales acuáticos, tanto en aguas saladas como en aguas dulces.

En el Cuadro 1 se muestra la evolución histórica del sector pesquero mexicano; con algunos datos relevantes en torno a la producción, tratados y apoyos que ha tenido, mostrando tasas de crecimiento favorables en la producción en los últimos años a pesar de la insuficiente atención e importancia en comparación a otros sectores.
En la Figura 1 se puede observar el cambio sustancial del crecimiento de esta actividad, ya que en 1994 apenas se registra un crecimiento de alrededor de 20.000 toneladas, para los años posteriores después de la firma del Tratado de Libre Comercio con Estados Unidos y Canadá se nota un mayor dinamismo en la producción; llegando a alcanzar cifras de 160.000 toneladas en el año de 2008.

En el año 2007 México produjo entre 1.600 y 1.400 toneladas métricas de productos pesqueros y se caracteriza por ser un país con tendencias a la exportación, ya que su balanza de pagos en este sector es positiva, con un saldo de 520 mil dólares. La Organización para la Cooperación y el Desarrollo (OCDE, 2007) señala que este incremento en la producción se debe entre otras cosas a la ubicación geográfica de México y que

Cuadro 1. Evolución del sector pesquero en México.

Table 1. Evolution of the fishing industry in Mexico.

\begin{tabular}{|c|c|}
\hline Año & Contexto histórico \\
\hline 1976 & $\begin{array}{l}\text { Se destinaron apoyos al sector pesquero; ello fomentó el crecimiento de dicha actividad a tasas récord 21\% } \\
\text { anual, alcanzando 1,6 millones de toneladas en } 1982 \text {. }\end{array}$ \\
\hline 1980 's & $\begin{array}{l}\text { La política pesquera siguió las tendencias de promoción del sector (Banpesca y organización de } \\
\text { cooperativas). Fundamentalmente se generó crecimiento de las capturas de camarón, atún y sardina; a la } \\
\text { par, las embarcaciones pequeñas tuvieron un crecimiento explosivo. }\end{array}$ \\
\hline 1990 's & $\begin{array}{l}\text { Salinas de Gortari a la presidencia, privilegió el mercado y la actividad empresarial privada y tomó las } \\
\text { riendas del sector pesquero como preparación a la entrada del Tratado Libre Comercio de América del } \\
\text { Norte (TLCAN); con ello disminuyeron los programas de subsidios y apoyos. }\end{array}$ \\
\hline 1993-1994 & $\begin{array}{l}\text { La flota se multiplico hasta alcanzar las } 66.557 \text { embarcaciones, de las cuales } 3.228 \text { eran de gran calado y } \\
\text { las } 63.329 \text { restantes eran pequeñas balsas, concentrando la fuerza de explotación en las zonas costeras en } \\
\text { un } 95 \% \text { y sólo un } 5 \% \text { en las zonas de altamar. }\end{array}$ \\
\hline
\end{tabular}

Fuente: Gómez (2008).

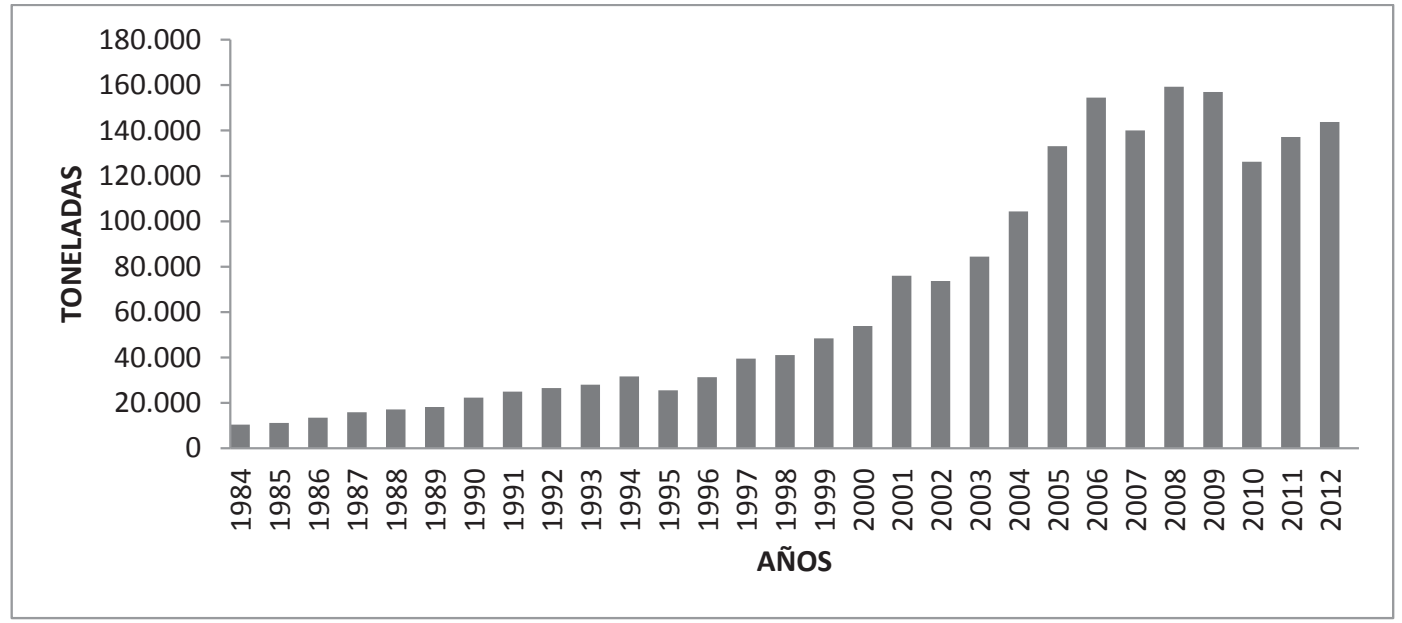

Figura 1.Producción pesquera en México. Fuente: Estadística Pesquera de la FAO, Producción en Acuicultura (2014a).

Figure 1. Fish production in Mexico. Source: Estadística Pesquera de la FAO, Producción en Acuicultura (2014a). 
tiene uno de los litorales más largos del mundo, con una extensión de $11.500 \mathrm{~km}$ (incluyendo sus islas mar adentro), de los cuales 73,7\% corresponde al Pacífico y 26,3\% al Golfo y el Caribe. La Zona Económica Exclusiva (ZEE) de México cubre casi tres millones de $\mathrm{km}^{2}$ con una plataforma continental que mide $358.000 \mathrm{~km}^{2}$, tiene 2,9 millones de hectáreas de aguas continentales, de las cuales 1,6 millones de hectáreas son lagunas y aguas costeras que resultan muy adecuadas para actividades acuícolas.

La Comisión Nacional de Acuacultura y Pesca, así como la Secretaría de Agricultura, Ganadería, Desarrollo Rural, Pesca y Alimentación (CONAPESCA-SAGARPA, 2014) comentan que en México, existe un importante desarrollo industrial adoptado desde hace más de 20 años, sin embargo, el desaprovechamiento en el sector primario sigue siendo un problema secular. Tal es el caso del sector pesquero, por lo que es necesario trabajar de manera conjunta con el sector productivo para optimizar el aprovechamiento del potencial que ofrece la pesca y la acuacultura.

No obstante, muchas de sus pesquerías han pagado caro el ascenso vertiginoso de la producción pesquera registrada a partir de los años sesenta (Gómez, 2008), ya que han desaparecido por falta de una adecuada planeación en su actividad productiva.

\section{Volumen de la producción}

En el 2011 las especies que formaron parte del volumen de producción pesquera en el país, en orden ascendente, fueron sardina industrial, sardina, camarón, atún, mojarra, anchoveta industrial, ostión, calamar, anchoveta, almeja, pulpo, carpa, jaiba y tiburón, entre una gran variedad de productos marinos. En ese año la mayor parte de la pesca se llevó a cabo en las costas del Pacífico. Por ejemplo, en dicho litoral se realizó la captura del 83\% del volumen de la producción pesquera total, en tanto que el litoral del Golfo y el Caribe participaron con el $14,4 \%$ y las entidades sin litoral contribuyeron con el 2,49\%.

De acuerdo con el último Censo Pesquero realizado en el 2013, el volumen de producción registrado en el año 2012 se concentra en el consumo humano directo, con 1.104.309 ton y en menor proporción el consumo humano indirecto 577.012 ton y uso industrial 6.177 ton. Este volumen de producción pesquera se captura fundamentalmente en el Litoral del Pacífico que representa el 82,6\%, y una menor proporción en el litoral del Golfo y el Caribe con 15,3\%. Se observa un crecimiento paulatino, la tendencia es positiva alcanzando en el año 2012 un volumen total de 1.687.498 toneladas.

El Valor Bruto de la Producción Pesquera (VBPP) a precios constantes ha tenido variaciones durante el periodo de 1987 al 2011. Las caídas son recurrentes de 1987 hasta el 2001 y una recuperación del 2003 hasta el 2010. La mayor caída se dio en el 2001 con 20,87\%. En el año 2011 el VBPP se estimó alrededor de 29.752 millones de pesos, según la Organización de las Naciones Unidas para la Agricultura y la Alimentación. La tasa más alta se observa en 1997 con 15,61 por ciento después de diferentes variaciones y una caída hasta el 2001; en 2002 tiene 8,04\%, nuevamente tiene una caída en el 2005 y se recupera al año siguiente con $11,62 \%$ y la última tasa de importancia fue en el 2010 con 7,14\%. Durante el periodo del 2003 al 2010 el sector pesquero ha tenido tasas de crecimiento positivas, reflejando cierta estabilidad, sin embargo para el año 2011 tuvo una tasa negativa de 11,31\% (FAO, 2014b).

El análisis anterior muestra fluctuaciones constantes, arrojando resultados tanto negativos como positivos, considerando la falta de atención en el sector podemos decir que han sido cifras "promedio". Lo que nos lleva a pensar que si implementamos las estrategias y medidas adecuadas para cada uno de los grupos que participan en el proceso de producción generaríamos mejores y mayores resultados. En este sector, predominan los pescadores de tipo tradicional, que de acuerdo a sus prácticas tecnológicas requieren mayor mano de obra para realizar sus actividades (Cuadro 2).

En tanto que la acuicultura es el conjunto de actividades, técnicas y conocimientos de crianza de especies acuáticas vegetales y animales. Es una importante actividad económica de producción de alimentos, materias primas de uso industrial y farmacéutico, y organismos vivos para repoblación u ornamentación (Román, 1992; Royero, 1993). La acuacultura es una actividad que abarca diversos aspectos y gama de especies, sistemas y prácticas. Es indudable que a pequeña escala, promueve el desarrollo socio-económico y cumple los objetivos de producción de alimentos, generación de ingresos, abasto de insumos a la agroindustria y provisión de empleo para los productores de escasos recursos.

La acuacultura tiene cada vez más mayor prominencia dentro de la producción pesquera. En México, dicha actividad también se ha instalado como una alternativa a la pesca tradicional, por ejemplo, se encuentra entre las primeras cinco naciones productoras de mojarra, tiburón cazón y ostión; en camarón, sardina y anchoveta, tiene el sexto lugar; en carpa, el séptimo, y en túmidos el octavo, según reportes de la (Rocha y Michel, 2001).

En los últimos años, debido a los problemas con los que se ha encontrado la pesca extractiva y al incremento de la demanda mundial de productos pesqueros, el sector ha provocado un crecimiento paulatino y firme de la acuacultura, la cual se encuentra en plena expansión, lo que debe ser aprovechado a través de incentivos y proporcionando recursos financieros para su competitividad (Hernández et al., 2009).

La acuacultura en México ha sido desarrollada en forma muy variada, en la mayoría de las regiones del País, utilizando diversas prácticas y sistemas de pro- 
Cuadro 2. Estructura del sector pesquero en México.

Table 2. Structure of the fishing industry in Mexico.

\begin{tabular}{ll}
\hline Definición & \multicolumn{1}{c}{ Pesca Artesanal } \\
\hline Embarcaciones & $\begin{array}{l}\text { Le lleva a cabo mediante el uso de técnicas y equipos menos sofisticados. } \\
\text { con autonomía de } 1 \text { día, de } 1 \text { a } 3 \text { toneladas de capacidad, con tripulación de } 2 \text { a } 4 \text { integrantes; no cuenta } \\
\text { con sistema de refrigeración para conservación del producto y la travesía es de corto tiempo }\end{array}$ \\
& $\begin{array}{l}\text { Elevado número de personas y acciones, que además desarrollan actividades colaterales como la confec- } \\
\text { ción y reparación de redes, construcción y mantenimiento de embarcaciones, reparación de motores. }\end{array}$ \\
\hline Productores & \multicolumn{1}{c}{ Pesca Industrial } \\
\hline Definición & $\begin{array}{l}\text { Es aquella que emplea embarcaciones de mayor tonelaje cuya autonomía permite realizar viajes de larga } \\
\text { distancia y duración. }\end{array}$ \\
Embarcaciones & $\begin{array}{l}\text { Eslora 22 metros; manga 6,88 metros; puntal 3,55 metros; calado, 2,50 metros; desplazamiento, 1,72 } \\
\text { toneladas métricas; velocidad máxima, 11 nudos (20,4 km/hr); autonomía de navegación de 21.000 } \\
\text { millas náuticas (38.892 kilómetros). }\end{array}$ \\
Productores & Empresas pesqueras
\end{tabular}

Fuente: Elaboración propia con datos de Villegas (2012), SAGARPA-CONAPESCA (2011).

ducción, desde intensivo, semi-intensivo y extensivo. En la acuacultura intensiva usan insumos de alta calidad y cantidad, permitiendo altos rendimientos productivos, la acuacultura extensiva puede a su vez emplear fertilizantes y alimentos suplementarios de calidad inferior y en menor cantidad, lo que resulta en menores rendimientos en producción pero con costos menores de producción.

México se encuentra actualmente entre los países de mayor producción acuícola en América. Para el año 2002 , se registró un ingreso de más de $\$ 3.309$ millones de pesos por acuacultura, producto de 45.853 toneladas de camarón blanco del pacífico, 91.434 toneladas de peces de agua dulce (mojarra, carpa, trucha, lobina y charal) y 48.878 toneladas de ostión (CONAPESCA, 2004).

La acuicultura ha venido a sustituir la producción pesquera por captura, en particular en lo que se refiere a producción de camarón, con lo que se han incrementado las exportaciones del crustáceo en términos generales y ha logrado acceder a mercados como la Unión Europea.

Podemos concluir que la importancia de la actividad pesquera acuícola, se reconoció al entrar en vigor la Ley General de Pesca y Acuacultura Sustentables (LGPAS) en el año de 2007 que establece que la actividad pesquera acuícola se eleva a rango de asunto de seguridad nacional, por lo que la federación, estados y municipios comparten responsabilidades en los planes de desarrollo del sector pesquero y acuícola, así como de inspección y vigilancia (Hernández et al., 2009).

\section{Balanza comercial}

En el transcurso de los años 2007-2013 las exportaciones ascendieron a 1.049 millones de dólares con 371 mil toneladas de pescados y mariscos en diversas presentaciones. Mientras que la importación de productos pesqueros alcanzó un valor 679 millones de dólares y un volumen de 215 mil toneladas de producto, logrando con esto un saldo positivo de 370 millones de dólares (CONAPESCA-SAGARPA, 2011). Por otra parte, en las importaciones se observa crecimiento considerable en los años 2011-2012 y en 2013 disminuyó nuevamente (Figura 2).

\section{Relevancia sociocultural y alimentaria}

La Comisión Nacional de Acuacultura y Pesca y la Secretaría de Agricultura, Ganadería, Desarrollo Rural, Pesca y Alimentación (CONAPESCA-SAGARPA, 2014), indican que la pesca en México tiene un valor económico, social y alimentario importante. Su desarrollo es fundamental no sólo por su incidencia en la soberanía alimentaria con productos de alta calidad y algunas especies con accesibles precios, sino por su aportación en mano de obra e ingresos que impulsan el desarrollo regional.

SAGARPA (2011), resalta que el fomento a la pesca sustentable que realiza el Gobierno Federal contribuye a generar una mayor competitividad del sector y reducir el impacto ambiental, lo que se refleja en mejores ingresos para los trabajadores de la industria pesquera y productos de calidad para los consumidores. En este 


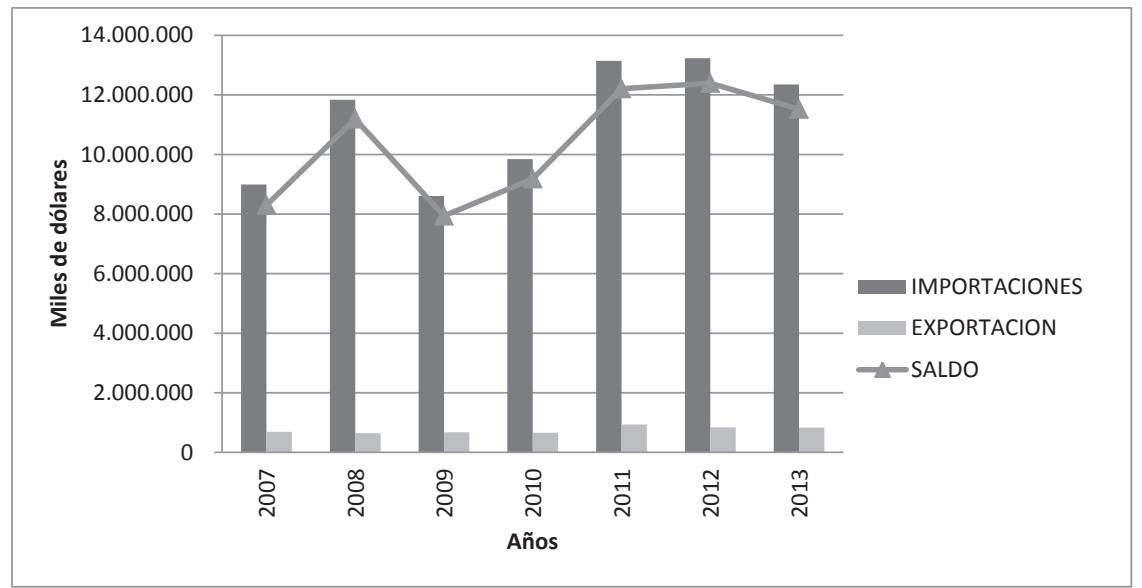

Figura 2. Balanza comercial de productos pesqueros 2007-2014 (miles de dólares). Fuente: Elaboración propia con datos de Banco de México (2014).

Figure 2. Trade balance of fisheries products, 2007-2014 (thousands of dollars). Source: Based on data from Banco de México (2014).

sentido es crucial para el sector que las medidas regulatorias sean practicadas y vigiladas cuidadosamente, es evidente que la reducción del impacto ambiental genera mayores recursos, por lo tanto forja un sector pesquero redituable. Sin mencionar que forma gran parte de nuestra identidad, al proveer de alimentación y ser uno de los sectores básicos para la medición de industrias de insumo, transformación y comercio.

Para el Colegio de Posgraduados en Ciencias Agrícolas (COLPOS, 2012), México ocupa el cuarto puesto en el Suministro de Energía Alimentaria (SEA), sólo superado por Cuba en América, Turquía y Túnez. Más importante es que los niveles de suministro superan las necesidades promedio por persona al día, estimadas en $2.357 \mathrm{kcal}$ para el periodo 2010-2012. Este promedio considera los requerimientos diferenciados por grupos de edad, género y ocupación. Es decir, que una distribución equitativa aseguraría un consumo adecuado de toda la población. Sin embargo, la desigualdad distribución del ingreso es un factor que actúa en contra.

En este sentido; se tendrá que dar especial importancia a dos aspectos relevantes: por un lado fomentar la cultura de consumo de pescado, ya que a pesar de contar con las condiciones físico geográficas que permiten un buen nivel de producción; la población mexicana no está acostumbrada a consumir pescado en forma permanente en su dieta; y por la otra parte es necesario revisar la política de precios de este tipo de productos, debido a que el consumo del mismo también se ve afectado por los altos precios que pagan los consumidores por el pescado y la difícil situación económica que vive México (CONAPESCA, 2008).

En un estudio comparativo realizado por FAO (2006), sobre seis países, incluido México, sobresale que coexisten extremos de la malnutrición: desnutrición, so- brepeso y obesidad, se observaron cambios en la dieta, similares a las modificaciones anotadas en la disponibilidad por grupos de alimentos. Las principales tendencias encontradas fueron: disminución en el consumo de cereales, raíces y tubérculos; reducción en el consumo de legumbres y semillas (con excepción de China); ningún cambio o aumento en el consumo de aceites y grasas comestibles; importantes aumentos en la ingesta de pescados, carne y pollo (excepto en India); creciente consumo de azucares y dulcificantes; y mayor consumo de frutas y hortalizas (excepto en China y Filipinas).

Por lo tanto el pescado es una fuente importante de alimentos de proteína animal. A nivel mundial, el suministro de peces comestibles per cápita aumento 9,9 (equivalente en peso vivo) en la década de 1960 hasta $18,4 \mathrm{~kg}$ en 2009. Cifras preliminares para 2011 indican que el consumo de pescado siguió aumentando hasta alcanzar los 18,8 kg. En México el consumo promedio en 2010 era de 10,6 kg (CONAPESCA, 2010).

México posee un gran potencial para la satisfacción de su seguridad alimentaria y la generación de desarrollo rural, tanto a nivel local como regional, mediante la generación de alimento para autoconsumo y para la comercialización de los excedentes, así como aprovechar su vasta diversidad y disponibilidad de zonas hidrológicas en todo el país. Sin embargo, el crecimiento del sector pesquero-acuícola deberá ser propiciado tomando en consideración el impacto que podría ejercer sobre el ambiente (FAO, 2014b).

\section{Ambiente}

La exagerada demanda de alimento provoca la sobreexplotación de los recursos pesqueros y conlleva problemas asociados, como son la captura incidental de 
especies no objetivo, el descarte de las especies sin valor comercial, y el deterioro ambiental, especialmente en las áreas costeras, que provoca la pérdida de hábitats y la contaminación. El Instituto Nacional de Pesca (INAPESCA, 2006) resalta que para reducir lo indicado anteriormente, se usan métodos de investigación en tiempo real, puntos de referencia, incertidumbre explicita y riesgo en la toma de decisiones. Un avance significativo para la evaluación de recursos pesqueros ha sido la implementación de sistemas de seguimiento por satélite en todas las embarcaciones mayores y menores. Sin embargo, la demanda excesiva ha generado un esfuerzo pesquero y tasas de aprovechamiento generalmente superiores a las que los recursos pesqueros pueden soportar.

Los ambientes costeros y oceánicos poseen una elevada riqueza biológica que contribuye a la megadiversidad y a la actividad económica de las zonas costeras y marinas del país. La riqueza natural de estas regiones atrae diversas actividades económicas como la agropecuaria, la extracción de hidrocarburos, el turismo, la industria, la acuacultura y la pesca; desafortunadamente, el desarrollo desordenado de éstas y otras actividades, así como el crecimiento poblacional han provocado graves problemas en ecosistemas altamente vulnerables (PND, 2007-2012).

En este sentido, específicamente en la producción acuícola es muy importante la conservación y rehúso del agua, ya que en los últimos años, es uno de los temas de mayor relevancia dentro de la acuicultura, debido a que es una de las estrategias fundamentales para incrementar la eficiencia en el uso del agua, utilizando los sistemas de recirculación del agua, además de que permite manipular la temperatura, el oxígeno disuelto, el fotoperiodo y la claridad del agua. Como consecuencia del control ambiental, la producción se ve aumentada, mejora la conversión alimenticia y los costos por tratamiento de enfermedades se ven reducidos, lo que maximiza los beneficios (Summerfelt et al., 2002).

\section{Empleo}

La pesca es una actividad que actualmente aporta el $0,8 \%$ del PIB nacional y emplea al menos el $1,3 \%$ de la población ocupada ( $20 \%$ del sector primario). Representa la actividad económica de mayor importancia, si no la única, en diversas regiones costeras del territorio nacional (CONAPESCA, 2010).

En el año 2007 la población dedicada a la pesca sumó un total de 283.656 personas, sin embargo en los años subsecuentes hasta 2012 disminuyó la población en un total de 266.380 personas. Por otra parte, se observa que en el rubro de población dedicada a la captura; es decir a la actividad pesquera, existieron disminuciones año tras año, en tanto que la población que participó en los sistemas de control (acuacultura) se incrementó de 30.418 en el año 2007 a 56.133 en el año 2012.

\section{Retos para México: Infraestructura, Financia- miento y Tecnología}

\section{Infraestructura}

La infraestructura pesquera es un elemento esencial para la operación eficiente del sector, registra importantes avances en el país, pues constantemente se realizan adecuaciones, adaptándose a los dinámicos cambios de la globalización. Dentro de la infraestructura sobresale el papel de la flota pesquera ya que constituye la herramienta fundamental de la actividad, hasta el 2011 hay 82.069 embarcaciones registradas, de las cuales 3.181 son de pesca de altura y 78.888 de pesca ribereña. En la pesca de altura se resalta el esfuerzo pesquero enfocado en la captura de camarón con 1.896 unidades con un tonelaje neto de entre 40 y 80 toneladas, una eslora promedio de 20 a 25 metros y una antigüedad de más de 30 años; de estas embarcaciones el $70 \%$ se encuentran registrada en el Litoral del Pacífico y el otro 30\% en el litoral del Golfo de México y Mar Caribe (CONAPESCA-SAGARPA, 2011).

El costo de mantenimiento y operación más significativo para los productores pesqueros y acuícolas, se encuentran relacionados con el consumo de diesel y gasolina. En la actividad de la flota pesquera mayor; el diesel representa entre el 37\% y $48 \%$ de la estructura de costos, en tanto que la gasolina llega a representar hasta el $72 \%$ en la pesca ribereña. En atención al mandato de la Ley de Energía para el Campo (LEC), publicada en diciembre de 2002, el Gobierno Federal ha instrumentado apoyos subsidiarios bajo diferentes mecanismos para la adquisición de energéticos (diesel, gasolina y energía eléctrica, principalmente) a precios de estímulo para los productores, a fin de impulsar la productividad y competitividad de la acuacultura y la pesca (SAGARPACONAPESCA, 2011).

A través del Proyecto Estratégico de Modernización de la Flota Pesquera Mayor, se busca reducir hasta en un $20 \%$ dichos costos, modernizar el sistema de refrigeración y conservación de los productos a bordo a fin de ofrecer al mercado nacional alimentos sanos y de calidad e incursionar en los mercados internacionales con presentaciones no tradicionales de mayor valor agregado (SAGARPA-CONAPESCA, 2011).

\section{Tecnología}

De acuerdo a lo anterior, y considerando los 30 años de antigüedad de la flota, podemos decir que es fundamental modernizar la infraestructura según la fracción III del artículo 2 que establece la Ley de Ciencia y Tecnología (LCyT), que señala que es necesario "Incorporar el desarrollo y la innovación tecnológica a los procesos productivos para incrementar la productividad y la competitividad que requiere el aparato productivo nacional" (Cisneros, 2011). 
En el 2010 la Secretaría de Agricultura, Ganadería, Desarrollo Rural, Pesca y Alimentación (SAGARPA) a través del Instituto Nacional de Investigaciones Forestales, Agrícolas y Pecuarias (INIFAP) y la Comisión Nacional de Acuacultura y Pesca (CONAPESCA), reconocieron la necesidad de tecnología en materia de genética y biotecnología por lo que firmaron dos Cartas de Intención para obtener de Japón transferencia de tecnología, asistencia técnica en biotecnología, capacitación para investigadores mexicanos, y asesoría para la comercialización de productos marinos (SAGARPA, 2010).

Por su parte el Instituto Nacional de Pesca (INAPESCA, 2012) plantea que conforme al Programa Sectorial de Desarrollo Agropecuario y Pesquero 2007-2012, el Gobierno Federal definió objetivos y líneas de acción claras para garantizar la disponibilidad de los recursos pesqueros y mejorar la calidad de vida de los agentes que participan en esta actividad, determinando, entre otras acciones, los métodos, técnicas y equipos de pesca de menor impacto ambiental y mayor valor agregado con el fin de hacer más eficiente y sustentable la captura marina. En este sentido, se espera que cumpliendo las políticas y lineamientos del programa, se puedan desarrollar adecuados proyectos de investigación, orientados para mejorar la tecnología en el sector pesquero de las diversas especies, de este modo incrementar la eficiencia operativa de la pesca que genere mayor producción y productividad para un mejoramiento en la calidad de vida de la población.

\section{Financiamiento}

Para el año 2011, el financiamiento al sector pesquero fue de 4.027 millones de pesos en donde Fideicomisos Instituidos en Relación con la Agricultura- Fondo de Garantía y Fomento para las Actividades Pesqueras (FIRA-FOPESCA) contó con una participación activa de 3.933 millones de pesos que se traduce en el $98 \%$ de financiamiento al sector, es decir que es un financiamiento que se obtiene fundamentalmente por vía interna; otras fuentes (Cuadro 3) estuvieron representadas por el Fondo Nacional de Apoyo para las Empresas de Solidaridad (FONAES) (CONAPESCA-SAGARPA, 2011).

El 75,9\% del financiamiento lo absorbieron las unidades dedicadas a la pesca silvestre, mientras que el $24 \%$ fue asignado a la acuacultura. Los principales usos a los que se destinó el financiamiento obtenido fueron para el funcionamiento, equipamiento o ampliación del negocio y la adquisición de insumos (mercancías, materiales, materias primas, etc.) en el mercado nacional. Actualmente se aplica el Programa de Apoyos Directos a la Pesca (PROPESCA), en 2014 opera con 100 millones de pesos para atender a 11,353 pescadores, busca desarrollar habilidades técnicas y aumentar la productividad y competitividad del sector.

\section{Chile productor pesquero-acuícola a nivel inter- nacional}

\section{Antecedentes}

A continuación se muestra en el Cuadro 4, una breve reseña histórica del sector pesquero, destacando que si bien se ha tratado de dar importancia al cuidado del ecosistema, Chile aún presenta grandes desafíos para mejorar sus sistemas de producción para que no genere más contaminación en el sector pesquero.

Chile junto con Perú y Ecuador, creó en 1952 la Comisión Permanente del Pacífico Sudeste (CPPS), y con ellos suscribió la Declaración de Santiago, tratado que establece una zona marítima de 200 millas de importancia económica y de conservación. La CPPS coordina y procura expresar una política marítima regional del pacífico sudeste. A este acuerdo, se incorporó Colombia con posterioridad. Chile, siempre interesado en participar en los convenios idóneos de conservación y administración pesquera, es integrante de otros tratados y convenciones. Lo cual quiere decir que el crecimiento de esta actividad se debe, entre otras cosas, al fomento de convenios comerciales, acuerdos y leyes que permiten la comercialización de sus productos con otros países.

Chile es un país pesquero y de acuicultura, cuyas principales fortalezas y ventajas comparativas radican

Cuadro 3. Financiamiento al sector pesquero por FIRA-FOPESCA por litoral y entidad federativa serie anual 2007-2012 miles de pesos.

Table 3. Financing of the fishing industry by FIRA-FOPESCA by coast and state, 2007 to 2012 annual series thousands of pesos

\begin{tabular}{lrrrrrr}
\hline & $\mathbf{2 0 0 7}$ & $\mathbf{2 0 0 8}$ & $\mathbf{2 0 0 9}$ & $\mathbf{2 0 1 0}$ & $\mathbf{2 0 1 1}$ & $\mathbf{2 0 1 2}$ \\
\hline LITORAL DEL PACÍFICO & 2.871 .472 & 2.939 .510 & 3.061 .815 & 3.108 .406 & 3.585 .094 & 4.511 .217 \\
LITORAL DEL GOLFO Y CARIBE & 266.285 & 309.939 & 250.607 & 262.589 & 247.559 & 312.189 \\
ENTIDADES SIN LITORAL & 17.155 & 33.950 & 19.247 & 36.546 & 100.786 & 165.241 \\
\hline
\end{tabular}

Fuente: CONAPESCA-SAGARPA (2011) 
Cuadro 4. Evolución del Sector Pesquero en Chile.

Table 4. Evolution of the fishing industry in Chile.

\begin{tabular}{ll}
\hline Año & \multicolumn{1}{c}{ Contexto histórico } \\
\hline $\mathbf{1 8 9 2}$ & $\begin{array}{l}\text { El Gobierno decretó la prohibición de la caza de lobos marinos y de nutrias, por haberse llegado al borde } \\
\text { de su extinción después de la depredación de los cazadores extranjeros }\end{array}$ \\
$\mathbf{1 9 7 6}$ & $\begin{array}{l}\text { Se caracterizó por un estímulo a la pesca industrial y artesanal, tarea que fue asumida por el Instituto de Fo- } \\
\text { mento Pesquero (IFOP) }\end{array}$ \\
$\mathbf{1 9 7 6}$ & $\begin{array}{l}\text { El Ministerio de Agricultura, era el responsable del sector pesca. Posteriormente el Ministerio de Economía, } \\
\text { creándose la Subsecretaría de Pesca (Subpesca), específicamente la División de Protección Pesquera del Servi- } \\
\text { cio Agrícola y Ganadero. }\end{array}$ \\
$\mathbf{1 9 7 8}$ & \begin{tabular}{l} 
En 1978, el Servicio Nacional de Pesca (Sernapesca) agregaron Acuicultura a su nombre. \\
Se promulgó la modificación de la Ley de Pesca y Acuicultura, dirigida a la sostenibilidad y recuperación de las \\
\hline
\end{tabular} \\
\hline
\end{tabular}

Fuente: ODEPA (2014)

en la alta productividad del ecosistema marino existente frente a las costas y dentro de su Zona Económica Exclusiva (200 millas náuticas). La pesca industrial en Chile se inició en la década de los 50 con barcos de madera de 40 a 50 toneladas. Desde 1974, Chile ha tenido una rápida evolución pasando de ser un país con pocas pesquerías costeras, con desembarque de 1,1 millones de toneladas, a convertirse en una de las principales naciones pesqueras, con operaciones en toda su Zona Económica Exclusiva y en importantes sectores de alta mar (SUBPESCA, 2014).

En el Cuadro 5 se señalan los tipos de pesca que se practican en Chile, principalmente de tipo artesanal y la industrial, donde se refleja que la pesca de tipo artesanal es la que más predomina y que sus actividades son de tipo tradicional generando un mayor número de empleos.

En Chile la concentración de la economía pesquera ha amenazado con diluir los límites que han separado a la pesca artesanal de la industrial (Cuadro 5). En el contexto de esa tendencia, se ha propuesto introducir en Chile un régimen de primera venta en subasta de las capturas de origen artesanal. El objeto de esta iniciativa es permitir que la pesca artesanal mantenga altos niveles de independencia respecto de las compañías pesqueras industriales, obtener mejores precios por sus ventas, y dotar al sector de mejores condiciones de transparencia en la comercialización de la pesca (UNAP, 2007).

\section{Volumen de la producción}

Actualmente, el país sudamericano genera el 48,5\% de la producción acuícola total de América Latina, lo que lo convierte en la principal región productora del continente y el Caribe. En 2007 de las más de 1.750.000 toneladas de productos acuícolas que se generaron ese año, 853.140 se produjeron en Chile, donde la producción de salmón acaparó la mayor proporción, con cerca del 90\% y que principalmente está orientado a la exportación (Industrias Pesqueras, 2010).

El territorio chileno produce casi una tercera parte (31\%) del salmón que se cultiva en todo el mundo. Es el segundo mayor productor sólo por detrás de Noruega, que genera el 33\%, y en los últimos años se ha convertido en el mayor exportador de salmónidos a Japón y Estados Unidos. Las claves del notable desarrollo de la salmonicultura en Chile, que se remonta a la década de 1980, hay que buscarlas, en las apropiadas y ventajosas condiciones naturales que ofrece el país, sus costos de producción, el entorno macroeconómico global y el apoyo público y privado dirigido a la fase productiva ${ }^{1}$.

Por su parte, la Pontificia Universidad Católica de Valparaiso (PUCV, 2010) indica que Chile es el quinto país del mundo en producción pesquera, el séptimo en producción acuícola y el séptimo mayor exportador del mundo de productos pesqueros. En un contexto general las exportaciones pesqueras chilenas, han aportado a la economía nacional, cifras que van entre el $12 \%$ y $13 \%$ en forma relativamente constante a través de la última década (FAO, 2014a).

Hasta aquí hemos observado que la economía chilena es un claro ejemplo de liberación comercial por los resultados "exitosos" en la aplicación de políticas de corte liberal, que tienden a la llamada especialización productiva que descansa en la producción fundamentalmente primaria, de aquellos productos tradicionalmente demandados por el mercado internacional, y en los que Chile venía participando desde la primera revolución industrial, además de otros derivados de la celulosa, cobre, papel, así como actividades pesqueras, para participar en el mercado mundial. El peso del

\footnotetext{
Véase: El Potencial acuicultor de América Latina, en www.industriaspesqueras. com, consultado el 25 de agosto de 2014
} 
Cuadro 5. Estructura del sector pesquero en Chile.

Table 5. Structure of the fishing industry in Chile.

\begin{tabular}{|c|c|}
\hline \multicolumn{2}{|r|}{ Pesca Artesanal } \\
\hline Definición & $\begin{array}{l}\text { Actividad pesquera extractiva realizada por personas naturales en forma personal, directa y habitual y, } \\
\text { en el caso de las áreas de manejo, por personas jurídicas compuestas exclusivamente por pescadores } \\
\text { artesanales, inscritos como tales. }\end{array}$ \\
\hline Embarcaciones & $\begin{array}{l}\text { El } 71,2 \% \text { de las embarcaciones entran en la categoría de botes, } 20,6 \% \text { son lanchas menores, mientras } \\
\text { que las lanchas medias y mayores giran en torno al } 4 \% \text { de cada una. }\end{array}$ \\
\hline Productores & $\begin{array}{l}\text { Un pescador artesanal es aquel que se desempeña como patrón o tripulante en una embarcación arte- } \\
\text { sanal. Son oficialmente, } 75.321 \text { personas los que sumados a sus familias permiten estimar en torno a } \\
\text { las } 200.000 \text { personas a quienes dependen directamente de esta actividad. }\end{array}$ \\
\hline \multicolumn{2}{|r|}{ Pesca Industrial } \\
\hline Definición & $\begin{array}{l}\text { Se considera a la actividad extractiva realizada por embarcaciones de una eslora superior a los } 18 \\
\text { metros, con sistemas de pesca tecnologizados. }\end{array}$ \\
\hline Embarcaciones & $\begin{array}{l}\text { Las naves se encuentran clasificadas en la categoría Industrial con rangos de Eslora (longitud máxima) } \\
\text { que van entre los } 16 \text { y los } 35 \text { metros, con un promedio de } 21,8 \mathrm{~m} \text {. para las embarcaciones de hombres } \\
\text { y con un promedio de } 23,8 \mathrm{~m} \text {. para las naves de mujeres. }\end{array}$ \\
\hline Productores & $\begin{array}{l}\text { En el Registro Nacional Pesquero Industrial figuran un total de } 136 \text { personas naturales de las cuales } \\
\text { un } 92 \% \text { corresponde a hombres inscritos y un } 8 \% \text { a mujeres. Poseen } 3 \text { naves lo que significa un } 20 \% \\
\text { del total de naves que están efectuando capturas en la actualidad. }\end{array}$ \\
\hline
\end{tabular}

Fuente: Elaboración propia con datos de SERNAPESCA (2014), SUBPESCA (2014) y CENDEC (2010)

sector exportador chileno da cuenta de una modernización y reestructuración productiva tal que permite ventas al exterior desde los años ochenta por más del $30 \%$ del PIB del país. El éxito de la economía chilena según Castillo (1997) se enfoca en una fase denominada de especialización productiva.

\section{Balanza Comercial}

En el contexto económico nacional, el sector pesquero y de la acuicultura tiene una participación promedio del 7,3\% de las exportaciones nacionales entre los años 2003 y 2009. En cuanto al PIB, entre los años 2003 y 2008, el sector aportó un promedio de 1,3 \% (PUCV, 2010).

Asimismo, Chile presenta una posición privilegiada en cuanto a sus exportaciones, logrando en 2008, un valor superior a los US $\$ 4.110$ millones, y comprometiendo un total de 1.356.000 toneladas. Cabe destacar que del valor total de las exportaciones, el $65,5 \%$ corresponde al sector acuicultor (SUBPESCA, 2014).

El Servicio Nacional de Pesca y Acuicultura de Chile indica que el país se encuentra entre los primeros diez países a nivel mundial, tanto en desembarque pesquero como en producción acuícola, especialmente en lo referido a la salmonicultura. En el año 2012, el total de las exportaciones de productos pesqueros y acuícolas bordearon los 4.600 millones de dólares, lo que refleja el aporte de esta actividad a la economía del país

\section{Ambiente}

Aunque Chile tiene notable producción pesquera principalmente de salmón, aún falta profundizar en las peligrosas consecuencias medioambientales de la industria salmonera. La cual ha provocado crecimiento de algas y contaminación del mar. Han utilizado de forma indiscriminada antibióticos, acaricidas, fungicidas y otros químicos para prevenir plagas, esto ha producido una mutación en un pequeño crustáceo que se instala bajo las escamas de los peces, el Caligus o piojo de mar (García-Moreno, 2005). Hay estudios de laboratorio que demuestran que el Caligus ya se ha convertido en una especie superior, como si fuera otra especie, absolutamente resistente a todos los químicos que se conocen. Esto ha generado un problema, no sólo en esta industria, sino en todas las especies de la zona (García-Moreno, 2005).

\section{Empleo}

En términos geográficos, esta actividad se ha desarrollado fundamentalmente en áreas rurales, lo que ha generado un importante crecimiento económico de algunas zonas de extrema pobreza de Chile. Los más de 2.400 centros de cultivo proporcionan empleo directo a 140 mil personas: 1.900 trabajadores de naves industriales, 70 mil pescadores artesanales, 43 mil trabajadores de planta de proceso y 27 mil trabajadores de la acuicultura (PUCV, 2010). 


\section{Inversión en tecnología e infraestructura}

Este desarrollo, ha sido posible debido a la permanente incorporación de inversiones tecnológicas de primer nivel en todas las fases del proceso pesquero: flota, descarga, transporte, y la construcción de modernas fábricas e instalaciones frigoríficas de diversa índole. Durante el período comprendido entre 2000-2008, se totalizaron inversiones tanto en el área extractiva como acuícola, por un monto de \$2.678 millones. Además, las empresas han reforzado la fase de comercialización, lo que ha permitido desarrollar nuevos productos y mercados. El crecimiento de la actividad pesquera dio origen a una activa demanda de embarcaciones con tecnología cada vez más especializada (SUBPESCA, 2014).

El Fondo de Fomento para Pesca Artesanal (2014), resalta que en el ámbito de la capacitación y asistencia técnica, el Fondo prioriza las actividades de capacitación, focalizadas hacia la diversificación productiva, transferencia de tecnología y conocimientos que permitan desarrollar las actividades pesqueras de extracción, manipulación, procesamiento, cultivo y comercialización según los requerimientos de las normativas vigentes de calidad de los mercados, como también hacia actividades orientadas al incremento de la eficiencia extractiva pesquera, a través de la introducción de tecnologías apropiadas e innovadoras de extracción. Podemos entonces afirmar que los aumentos en el volumen de producción que tiene Chile se deben justamente a las inversiones en desarrollo tecnológico y en su infraestructura.

\section{Discusión}

En un diagnóstico de la pesca y la acuicultura elaborado por el (INAPESCA, 2012) se concluyó que la pesca y la acuacultura de México necesitan basarse en investigaciones realizadas en campos nuevos del conocimiento. La complejidad de los fenómenos naturales y sociales obliga a buscar otras formas de interpretación de la realidad. Se requieren la interdisciplina y la transdisciplina para abordar los problemas de hoy. La orientación de la pesca y la acuacultura hacia la sustentabilidad necesita investigación sólida y transferencia tecnológica validada. La necesidad de realizar un aprovechamiento cuidadoso y ordenado de los recursos naturales para generar mayores beneficios convierte en prioritaria la investigación científica y tecnológica para atender la pesca y la acuacultura de tal manera que se desarrollen en condiciones de sustentabilidad (Cisneros, 2011).

Es importante mencionar que la FAO, a partir de 1995, adoptó el Código Internacional de Conducta para la Pesca Responsable para orientar a sus ejecutores hacia las buenas prácticas de pesca. Es determinante contar para cada recurso con un plan de manejo ya que resultan la vía legal para encaminarnos al uso sustentable de los recursos pesqueros mediante la apropiada extracción de los organismos acuícolas y sus actividades relacionadas en términos biológicos, tecnológico, económicos, sociales, ambientales y comerciales, donde se promueve la conservación, gestión y desarrollo de las pesquerías con concordancia del ecosistema y el mantenimiento de la biodiversidad asociada (CONAPESCA- SAGARPA, 2008).

Se observa que México, a pesar de contar con más litoral que Chile, no tiene la suficiente producción lo que nos indica problemas en el ámbito de la fase de captura a nivel técnico, por lo que también se requiere incrementar los procesos de capacitación, infraestructura y desarrollo tecnológico en esta fase; elaborar un diagnóstico sobre la existencia y vida útil estimada de la infraestructura de uso común para poder determinar la demanda de nueva infraestructura y las prioridades de rehabilitación y mantenimiento; evaluar las condiciones de los cuerpos lagunares costeros para establecer necesidades de dragado y escolleras; e incrementar el abasto interno de productos pesqueros ya que se cuenta con un suministro de alimento de origen marino bajo ${ }^{2}$.

Si bien en 2007 se promulgo la Ley General de Pesca y Acuacultura Sustentables, que contiene nuevos elementos y prescribe líneas concretas de acción para las actividades pesqueras y acuícolas nacionales, es necesario dar seguimiento al Programa Nacional de Investigación Científica y Tecnológica en Pesca y Acuacultura (PNICTPA) que debe ser operado por la Red Nacional de Información e Investigación en Pesca y Acuacultura (RNIIPA) (Cisneros, 2011).

México ha tenido avances sustanciales en acuerdos y convenios, sin embargo requiere fomentar este tipo de instrumentos que permitan ampliar acuerdos comerciales con otros países; el país tiene que considerar como estrategia secular su adhesión a futuros acuerdos, códigos y comisiones internacionales, reactivando el compromiso, relevancia e impacto que tuvo inicialmente al considerarse como uno de los promotores del orden jurídico en el mar. Cabe destacar que la integración a nuevos acuerdos no implica que exista desarrolo inmediato y sustancial, se deben contemplar otras variables de carácter político-económico, y en sinergia lograr un sector pesquero acuícola óptimo.

En México, la actividad pesquera al igual que otras actividades del sector agropecuario presenta fuertes problemas en el procesamiento y comercialización, lo que debe ser fomentado principalmente con inversión

\footnotetext{
Dragado: Se define como el conjunto de operaciones necesarias para la extracción, el transporte y el vertido de materiales situados bajo el agua, ya sea en el medio marino, fluvial o lacustre. Escolleras: Consiste en la extensión en general en forma de manto o repie, de piedras relativamente grandes sobre un talud preparado, formando una capa compacta, bien graduada y con un mínimo de huecos (CONAPESA- SAGARPA, 2008).
} 
en el ámbito de las asesorías en formulación de proyectos y nuevas tecnologías en genética, biotecnología y manejo del producto. México aún está muy por debajo de las demandas de inversión que requiere esta actividad, ya que como se comentó la infraestructura requiere ser renovada.

Para el caso de Chile, su posición en el mercado internacional ha sido a costa de degradar el medio ambiente y a una mayor polarización social de sus sectores productivos que se han visto afectados con la implementación de sistemas de producción intensivos en uso de tecnología, desplazando a mano de obra.

Si bien a 24 años del inicio de la acuicultura intensiva en Chile se han constatado importantes beneficios económicos, se reconoce que todavía es necesario realizar avances en el aprovechamiento sustentable del ambiente, debido a que el impulso a la producción acuícola industrial ha dado prioridad a la productividad que al cuidado del ecosistema, siendo uno de los sectores que más contaminación genera. Asimismo se han obtenido progresos con la promulgación de varios reglamentos, los que sin embargo presentan el desafío de ser completamente implementados.

De acuerdo al análisis general anterior, podemos concluir que Chile es una potencia pesquera y acuícola en América Latina, gracias a la incorporación constante de tecnología en el sector, además de la implementación innovadora en el marco regulatorio destacando los límites máximos de captura, reduciendo notablemente capturas incidentales y de esta forma optimizando sus recursos. La pesca y la acuicultura chilenas han trabajado arduamente hasta convertirse en un sector estratégico para la economía del país, sin embargo ello también ha afectado a otros sectores sociales que sustentan mayoritariamente una producción de tipo artesanal.

Chile comparte similitudes con México, por lo que es posible realizar un análisis comparativo de la actividad pesquera de ambos países, y conocer la estrategia de Chile para potencializar su sector pesquero acuícola en los primeros lugares del mercado internacional. Cabe resaltar que a pesar de la ventaja de la ubicación geográfica de la que dispone México, Chile cuenta con un sector más sólido y redituable.

La actividad pesquera acuícola en México presenta diversas problemáticas, sin embargo sobresale el bajo desarrollo tecnológico, lo cual le afecta en su desenvolvimiento económico. Como lo plantea Schumpeter es necesario que las empresas encuentre ese desenvolvimiento económico porque esto les ayuda hacer competitivas ante sus principales competidores, para ello se requiere la innovación, desarrollo de tecnología y un cambio socio-cultural que impulse en forma decida este sector. Asimismo, el sector pesquero acuícola tiene que fomentar una cultura emprendedora para que su crecimiento sea eficaz dentro del mercado. En México el sector pesquero acuícola aún no ha tenido suficien- te apoyo en la introducción y aplicación de tecnologías debido a los limitantes en inversión y a que no se han entendido los beneficios que aporta la adopción de tecnologías en el marco de una mayor competencia a nivel internacional.

En cambio Chile a pesar de que tiene un lugar privilegiado en el comercio internacional de productos pesqueros acuícolas, requiere en suma atender los problemas de contaminación y atender con programas las diferentes demandas sociales y económicas de su población.

Para resolver algunas problemáticas señaladas, así como para cumplir los retos planteados es menester: reducir la pesca ilegal, limitando a las embarcaciones al marco regulatorio establecido, implementando un esquema de control y calidad; fomentar el consumo del pescado, con apoyo de programas y políticas públicas; hacer más competitivos los productos y así buscar nuevos mercados; obtener cierto número de embarcaciones que permita producir una cantidad competitiva reduciendo costos y maximizando utilidades; modernizar la flota pesquera, pues al innovar las embarcaciones se reduce un $20 \%$ de costo, se optimizan recursos y se incursiona en forma más provechosa en el mercado internacional; así como desarrolla y diseñar nuevos formas de presentar el producto, crear una campaña de publicidad para fomentar el consumo, y establecer estrategias claras de distribución.

\section{CONCLUSIONES}

El sector pesquero acuícola de México requiere hacer cambios sustanciales, modificar la situación de descuido y marginación en el que se encuentra principalmente la pesca de tipo tradicional, ya que la pesca de tipo industrial si bien aún presenta retos tecnológicos importantes que tienen que cubrir, debe de atender prioritariamente a los productores tradicionales para que puedan tener mejores condiciones de empleo que les permita cambiar sus condiciones de vida, por lo que es necesario apoyar con servicios, tecnología e infraestructura que requiere ser renovada.

A la pesca y la acuicultura en México como actividad económica-social, no se le está dando la debida importancia, ya que hacen falta programas y financiamiento gubernamentales. Y no solo como actividad, si no como base alimentaria en la dieta de los mexicanos. Estrategias, medidas, programas y financiamientos sólidos, implementados por el gobierno son poco útiles mientras el consumo interno no se incremente. Por sus cualidades nutricionales puede ser adoptado como una estrategia para cambiar el perfil alimentario del mexicano, además de disminuir problemas de salud como la obesidad y la desnutrición (Avilés y Vásquez, 2006).

La explotación racional de los recursos sustentables de la pesca y acuacultura, permitirá al país contar con 
una actividad productiva estratégica para su desarrollo a largo plazo, generando alimento, empleo, divisas y desarrollo regional. Asia será el principal demandante de alimentos a nivel mundial, en tanto que América Latina podrá convertirse en un proveedor importante, contexto en el que México podrá destacar como abastecedor confiable, según lo indican datos de la Organización de las Naciones Unidas para la Alimentación y la Agricultura (FAO) y de la Organización Mundial de Comercio (OMC) (Astorga, 2010). Tomando en cuenta una proyección sobre el crecimiento de la población mundial, se estima que al menos un adicional de 40 millones de toneladas de alimento de origen acuático, será requerido para el año 2030 para mantener el consumo per cápita (NACA/FAO, 2001).

El caso de Chile es un claro ejemplo de que a pesar de que cuenta con menos litoral, obtiene mejores resultados en la extracción, aprovechamiento y comercialización de productos del mar, debido a la política de apoyos en infraestructura, capacitación, tecnología e inversión; lo que le ha permitido penetrar cada vez más en el mercado internacional.

El actual crecimiento del sector pesquero acuícola en Chile es resultado de la aplicación de políticas públicas neoliberales, sustentadas en fuertes procesos de inversión en tecnología e infraestructura que agiliza la apertura comercial, y que además aprovecha las condiciones físico geográficas, y de mano de obra que permiten una organización de la producción orientada a cubrir prioritariamente las demandas y requerimientos del mercado externo.

Tanto México como Chile están apostando a fomentar la producción pesquera-acuícola, orientada principalmente al mercado externo; como resultado de la apertura comercial; pero que en términos sociales está afectando la vida socio cultural de sus habitantes, ya que se está dando prioridad al crecimiento económico dejando de lado el desarrollo y mejor calidad de vida de sus habitantes.

\section{REFERENCIAS}

ASTORGA L. 2010. Firman México y Japón acuerdos para exportación de productos pesqueros y acuícolas durante el Foodex Japan 2010, Financiera Rural, 15 de Marzo del 2010, en http://www.financierarural.gob.mx, [Consulta: 12 de junio de 2014].

AVILÉS, Q.S, VÁSQUEZ, H.M. 2006. Fortalezas y debilidades de la acuacultura en México. En: Guzmán, P., Fuentes D. (Eds.), Pesca acuacultura e investigación en México. Cámara de Diputados. Comisión de Pesca. CEDRSSA. México.

BANCO DE MÉXICO. 2014. Balanza de productos agropecuarios, en http://www.banxico.org.mx, [Consulta: 12 de junio de 2014].
CENDEC. 2010. Situación del fomento productivo en la pesca artesanal en Chile, en http://www.cendec.cl/ [Consulta: 18 de octubre de 2014].

CISNEROS, M.A. 2011. La RNIIPA: plataforma para el desarrollo acuícola y pesquero de México. Ciencia Pesquera 19(2): 77-84.

COLEGIO DE POSTGRADUADOS (COLPOS). 2012. Panorama de la seguridad alimentaria y nutricional en México, en http://www.colpos.mx, [Consulta: 12 de junio de 2014].

COMISIÓN NACIONAL DE PESCA Y ACUACULTURA-CONAPESCA. 2010. Políticas de ordenamiento para la pesca y acuacultura en el marco del programa rector de pesca y acuacultura, en http://www.gbcbiotech.com, [Consulta: 09 de junio de 2014].

CONAPESCA. 2004. Sector pesquero en México en Anuario estadístico de pesca 2003 en http://www.siap.gob.mx/ [Consultado 15 de diciembre de 2014].

CONAPESCA-SAGARPA. 2008. Diagnóstico y planificación regional de la pesca y acuacultura en México, Resumen Ejecutivo, en http://www.conapesca.sagarpa.gob.mx, [Consultado 13 de junio de 2014].

CONAPESCA-SAGARPA. 2011. Anuario Estadístico de Acuicultura y Pesca, en http://www.conapesca.sagarpa.gob.mx, [Consulta: 12 de junio de 2014].

CONAPESCA-SAGARPA. 2014. Alianza con el Legislativo, fundamental para impulsar al sector pesquero y acuícola, en http://www.conapesca.sagarpa.gob.mx, [Consulta: 12 de junio de 2014].

FONDO DE FOMENTO PARA LA PESCA ARTESANAL. en http://www.fondofomento.cl [Consulta: 10 de junio de 2014].

GACITÚA, S., PIZARRO, F. 2007. Universidad Arturo Prat, Departamento de ciencias del Mar "Situación de la Pesca Artesanal en Chile: institucionalidad, perspectiva y futuro en http://www.unap.cl/ [Consultado 13 de junio de 2014].

GARCÍA-MORENO, F. 2005. Salmones en Chile. El negocio de comerse el mar. Colección soberanía alimentaria de veterinarios sin fronteras. Documento 4. Fundación Kontrast.

GITMAN, L. 2003. Principios de Administración Financiera. Pearson, Educación. México.

GÓMEZ, J. 2008. México y sus recursos pesqueros ante el tratado de libre comercio. En: Presente y Futuro de los sectores ganadero, forestal y de la pesca. (Eds.), El Colegio de México, A. C., Universidad Autónoma de Chapingo.

HERNÁNDEZ, C., AGUIRRE, G., LÓPEZ, D. 2009. Sistemas de producción de acuacultura con recirculación de agua 
para la región norte, noreste y noroeste de México".

INAPESCA. 2006. Sustentabilidad y Pesca Responsable en México, en http://www.inapesca.gob.mx, [Consulta: 10 de agosto de 2014].

INAPESCA. 2012. La innovación y desarrollo tecnológico pesquero como pieza clave de una pesca sustentable. Caso selecto: la pesquería de camarón en el Océano Pacifico mexicano, en http://www.inapesca.gob.mx, [Consulta: 10 de agosto de 2014].

INDUSTRIAS PESQUERAS. 2010. El potencial acuicultor de América Latina, en www.industriaspesqueras.com, [Consulta: 25 de agosto de 2014].

INSTITUTO NACIONAL DE ESTADÍSTICA Y GEOGRAFÍA. 2014. Boletín de información oportuna del sector alimentario, en http://www.inegi.org.mx, [Consulta: 11 de junio de 2014].

INSTITUTO NACIONAL DE ESTADÍSTICA Y GEOGRAFÍA (2013), El sector alimentario en México, en http:// www3.inegi.org.mx, [Consulta: 28 julio de 2014].

INSTITUTO NACIONAL DE ESTADÍSTICA Y GEOGRAFÍA. 2010. Balanza comercial de México, en http://www.inegi.org.mx, [Consulta: 10 de junio de 2014].

LARA-LARA, J.R. 2008. Los ecosistemas costeros, insulares y epicontinentales. En: Capital natural de México, vol. I: Conocimiento actual de la biodiversidad. Conabio, México, en http://www.biodiversidad.gob.mx, [Consulta: 12 de junio de 2014].

MÉNDEZ, J.S. 1998. El neoliberalismo en México: ¿éxito o fracaso? Revista de Contaduría y Administración 199: 6574.

MITCHAM, C. 1994. Thinking through Technology, the Path between Engineering and Philosophy. En: Osorio, C. (Ed.), Enfoques sobre la Tecnología en: http://www. cneq.unam.mx/ [Consulta: 21 de octubre de 2014].

MODIGLIANI, F. MILLER, Y. 1958. The Cost of Capital, Corporation Finance and the Theory of Investment. American Economic Review 48(3): 261-297.

MONTOYA, 0. 2004. Schumpeter, innovación y determinismo. Sciatia et Technica. 25: 209-213.

NACA/FAO. 2001. Aquaculture in the third millennium. In: Subasinghe R. P., Bueno P.B., Phillips, M.J., Hough, C., McGladdery, S.E., Arthur, J.R. (Eds.), Technical proceedings of the conference on aquaculture in the third millennium, Bangkok, Thailand and Roma.

ODEPA MINISTERIO DE LA AGRICULTURA. 2014. Sector pesquero: evolución de sus desembarques, uso y exportación, en http://www.odepa.cl, [Consulta: 12 de junio de 2014].
ORGANIZACIÓN DE LAS NACIONES UNIDAS PARA LA ALIMENTACIÓN Y LA AGRICULTURA (FAO). 2006. The Double Burden of Malnutrition Case studies from six developing countries. Food and nutrition paper 84, Rome.

ORGANIZACIÓN DE LAS NACIONES UNIDAS PARA LA ALIMENTACIÓN Y LA AGRICULTURA (2014a), Estadística Pesquera de la FAO, Producción en Acuicultura 2014, en http://www.fao.org, [Consulta: 11 de julio de 2014].

ORGANIZACIÓN DE LAS NACIONES UNIDAS PARA LA ALIMENTACIÓN Y LA AGRICULTURA. 2014b. Visión general del sector acuícola nacional en: http://www.fao.org, [Consulta: 12 de junio de 2014].

ORGANIZACIÓN PARA LA COOPERACIÓN Y EL DESARROLLO (OCDE). 2007. Política agropecuaria y pesquera en México. Logros recientes continuación de las reformas, en http://www.oecd.org, [Consulta: 13 de junio de 2014].

OSORIO, C. 2002. Enfoques sobre la Tecnología, en http:// www.cneq.unam.mx, [Consulta: 21 de octubre de 2014].

PACEY, A. 1990. La cultura de la Tecnología, México: F.C.E, En: Osorio, C. (Ed.), Enfoques sobre la Tecnología en: http://www.cneq.unam.mx/ [Consulta: 21 de octubre de 2014].

PONTIFICIA UNIVERSIDAD CATÓLICA DE VALPARAÍSO (PUCV). 2010. http://ucv.altavoz.net, [Consulta: 10 de junio de 2014].

PND (2007-2012), Plan Nacional de Desarrollo, en pnd.calderon.presidencia.gob.mx, [Consulta: 25 de agosto de 2014].

QUINTANILLA, M. 1988. Tecnología: Un enfoque Filosófico En: Osorio, C. (Ed.), Enfoques sobre la Tecnología en: http:// www.cneq.unam.mx/ [Consulta: 21 de octubre de 2014]

ROCHA, G., MICHEL, G. 2001. Inseminación artificial, en: Gaceta Universitaria-Centro universitario del sur en http:// www.gaceta.udg.mx/ [Consultado 13 de junio de 2014].

ROMÁN, B. 1992. Peces Ornamentales de Venezuela. Fundación La Salle de Ciencias Naturales. Barcelona.

ROYERO, R. 1993. Peces Ornamentales de Venezuela. Cuadernos Lagoven, Lagoven, S.A. Caracas.

SAGARPA-CONAPESCA. 2011. El Sector Pesquero y Acuícola Logros 2007 a 2012, en http://www.conapesca.sagarpa.gob.mx, [Consulta: 18 de octubre de 2014].

SAGARPA. 2010. Firman México y Japón acuerdos que favorecen la producción y exportación de productos del campo y el mar, en http://sagarpa.gob.mx, [Consulta: 5 de junio de 2014].

SAGARPA. 2011. Pesca sustentable reduce impacto ambiental y aumenta competitividad del sector, en http://sagarpa. 
gob.mx, [Consulta: 10 de junio de 2014].

SHUMPETER, J. 1978. Teoría del desenvolvimiento económico. Quinta Reimpresión, Fondo de Cultura Económica, México.

SINGH, A., HAMID, J. 1992. Corporate financial structures in developing countries, Washington, "Technical paper 1", International Finance Corporation, World Bank.

SERNAPESCA. 2014. Pesca Artesanal, en www.sernapesca.cl [Consulta: 18 de octubre de 2014].

SUBPESCA. 2014. Pesca Industrial en Chile, en: http://www. subpesca.cl/ [Consulta: 18 de octubre de 2014].
SUBSECRETARÍA DE PESCA Y ACUICULTURA GOBIERNO DE CHILE. 2014. Chile Azul Principales Recursos Pesqueros y de Acuicultura, en http://www.subpesca.cl, [Consulta: 10 de junio de 2014].

SUMMERFELT, S., WILTON, G., ROBERTS, D., SAVAGE, T. 2002. Developments in recirculating systems for arctic char culture in North America. Proceedings of the fourth international conference on recirculating aquaculture, Roanoke, Virginia.

VILLEGAS, J. 2012. La pesca artesanal como estrategia de sobrevivencia: el caso de los pescadores en ciudad del Carmen, Campeche. Tesis Magister Facultad de Latinoamérica de Ciencias Sociales, sede México. 111p. 